\title{
Photometric Techniques for Exploring Multiple Populations in Clusters
}

\author{
Jay Anderson \\ Space Telescope Science Institute, 3700 San Martin Drive, Baltimore, MD 21218 USA
}

\begin{abstract}
The advent of the Hubble Space Telescope (HST) and the development of new photometric algorithms that take advantage of its stable observing platform above the atmosphere have allowed us to study the populations in globular clusters with very high precision.
\end{abstract}

Keywords. (Galaxy:) globular clusters: general, ultraviolet: stars, methods: data analysis

\section{Introduction}

Globular clusters have been studied intensely for decades both photometrically and spectroscopically, yet only in the past few years have we realized their true multipopulation nature. Spectroscopy has long hinted at non-singular abundances, but it was not until the high-precision photometry of unevolved main-sequence stars made possible by the Hubble Space Telescope (HST) that it was clear that the abundance variations indicated multiple (or gradual) formation episodes. This brief article will highlight the properties of HST that make these studies possible and the approaches that thus far have worked best to highlight the non-singular populations.

\section{The promise and challenges of HST}

Ground-based photometry has the advantages of well-sampled near-Gaussian PSFs and a large field of view (FOV), but must deal with variable seeing and resolution of $\sim 1^{\prime \prime}$ or worse. This makes crowding a significant issue for studying the faint stars near the cluster centers. HST has the benefits of being extremely stable, and also provides highresolution access to both the UV and IR and the large color baselines these filter systems make possible. The downside of HST is that we must deal with (1) an undersampled PSF, (2) large field distortion, and (3) a small FOV. Thankfully these challenging issues have been addressed with focused efforts at characterizing the detector and optimizing the approaches to photometry.

The HST PSF varies by $\pm 20 \%$ across the FOV of most detectors. Thankfully, HST's exquisite stability allows us to characterize this variation empirically once for all and then use it for all future analyses. Similarly, even though the PSF is undersampled, once we construct a model for the PSF from a well-observed starfield, we can use the PSF model to measure future observations. HST's detectors have a lot of distortion, but once the mapping from detector to sky coordinates has been done once, it can later be used to allow very precise differential astrometry.

Although HST's FOV is very small, it is compensated somewhat by its increased resolution. HST can study stars into the very centers of almost all clusters, so even within $3^{\prime} \times 3^{\prime}$ we can measure over a hundred thousand stars well. This has been a critical aspect of the multi-population study, since the second generation tends to be more concentrated than the first. HST's small FOV does, though, underline how important it is to combine space-based surveys of the central regions with ground-based surveys of the outer regions. 


\section{New approaches}

In addition to photometric techniques that have been optimized to the undersampled, yet stable, HST detectors, several additional approaches have been necessary to tease out the fine-scale structure present in globular-cluster CMDs. Recent papers by Antonino Milone exhibit all of the following.

Selective finding. Not all stars can be measured with the same accuracy. Some have nearby neighbors that prevent a good measurement. If we focus our efforts on the stars that are isolated, then we can improve our overall photometry. It is certainly true that bright stars are more likely to be considered "isolated" than faint stars, but the fine structure we are interested in concerns variations of color at the $1 \%$ to $5 \%$ level; these small variations introduce no bias in our finding stars on (say) the blue or red parts of the various branches. This selective-finding approach works for studying horizontal structure, but not vertical structure (luminosity-function-type analyisis).

Corrections for Differential Reddening. The focus above is on measuring accurate colors for stars in hopes of measuring fine structure of 0.01 magnitude in color across the main sequence (or other population sequences). Although globular clusters do not have much gas or dust, the lines of sight to these clusters often do, and differential reddening can introduce field-dependent shifts of 0.05 magnitude or more in color. The scale over which the reddening is coherent tends to be an arcminute or so. Typically we have hundreds of HST stars within each coherent patch, so it is possible to determine a fiducial sequence line within each region, then determine how to shift the sequence for each region along the reddening line to account for the variations in reddening.

Multiple Independent Observations. Even with the best possible static empirical models of the PSF, short-term and long-term variations in focus can introduce some small perturbations in the PSF that affect photometry at the $1 \%$ level. It can be hard to confidently average down multiple exposures to better than $1 \%$ if we do not have large dithers to allow us to assess such systematic spatial issues. Furthermore, we often must get photometry from the archive and must live with the limitations of the original observing plan. In this case, it can help to have more than one pair of filters, or multiple pointings with the same filter, so that we can assess whether a star on the blue side of the MS in one observation is also on the blue side in another.

Filter optimization. It was first thought that HST's WFC3 would help the multiple population programs because of its larger color baseline, providing coverage from the UV to the IR. But it turns out that there is a particular combination of three colors with HST that allow multiple populations to be identified trivially in color-color diagrams. Recent papers by Milone show what can be done with F275W, F336W, and a visible filter (say, F814W). This separation works well on all branches: MS, SGB, RGB, and HB. Once populations are separated in one color system, we can use other color systems to explore extremely fine structure in the CMD, structure that is well below the typical $1 \%$ level needed to make clean separations. Ground-based studies have investigated their own filter optimization by exploring the synergy of spectroscopy and photometry to determine which filters best allow known populations to be separated.

\section{What's next?}

The obvious next thing to do is to repeat the Sarajedini GC Treasury clusters with the optimal HST filter system; this will allow all clusters to have their multiple populations assessed. This will be combined with spectroscopy to give us details about each population. 